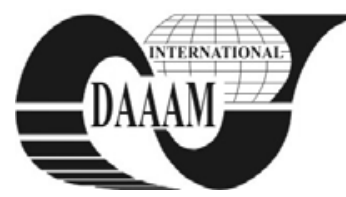

Annals of DAAAM for 2011 \& Proceedings of the 22nd International DAAAM Symposium, Volume 22, No. 1, ISSN 1726-9679 ISBN 978-3-901509-83-4, Editor B. Katalinic, Published by DAAAM International, Vienna, Austria, EU, 2011 Make Harmony between Technology and Nature, and Your Mind will Fly Free as a Bird Annals \& Proceedings of DAAAM International 2011

\title{
NEW METHOD OF ELECTROMAGNETIC DESIGN OF AC MACHINES
}

\author{
CIPIN, R[adoslav] \& VONDRUS, J[iri]
}

\begin{abstract}
The paper describes a new universal infinitesimal method which enables the analysis and synthesis of arbitrary AC motor windings. In this paper the new method is used to electromagnetic design of AC machines (the induction motor and the synchronous motors with permanent magnets) for arbitrary winding type. The method is based on the notion of the local angular density of conductors $n(\alpha)$. The function $n(\alpha)$ can be arbitrary, i.e. of continual or impulse character.

Key words: $A C$ motor winding, $A C$ winding design, density of conductor, winding factor
\end{abstract}

\section{INTRODUCTION}

The classical electromagnetic design of AC machines windings (induction motors and synchronous motors with permanent magnets) is based on total winding factor $k_{\mathrm{w}}$, see (Langsdorf, 1955), (Boldean \& Nasar, 2010) and (Pyrhonen et al., 2008). The total winding factor is given by product of the distribution factor $k_{\mathrm{q}}$ and chording factor $k_{\mathrm{y}}$. The total turn number $N$ in the winding of one pole-pair is then determined by a well-known equation:

$$
N=\frac{U_{r m s}}{2 \sqrt{2} \pi k_{\mathrm{w}} f B_{\text {max }} l r}
$$

where $B_{\max }$ is the amplitude of the flux density under the pole, $f$ is stator frequency, $U_{\text {rms }}$ is rms of phase voltage, $l$ is length of the stator sheaf, and $r$ is average radius of the air gap.

Well-known mathematical expressions for factors $k_{q}, k_{\mathrm{y}}$ are not universal as they have following limits:

- They are valid only for windings containing the same number of conductors in.

- They do not enable to analyze windings with "continually" (e.g. harmonic) distributed density of the conductors.

- They do not enable to analyze any irregular winding, e.g. fractional winding.

In following chapters, we will describe a new method, which does not include the above mentioned limits, and is universally useable for the analysis and synthesis of AC windings of any type. The very basic principles of this method were firstly published in (Nowotny \& Lipo, 1996).

\section{CONDUCTORS DENSITY OF THE WINDING}

Fig. 1. a) shows an example of the spatial distribution of conductors in a few slots, when respecting the current orientation.

The angle $\alpha$ is an electrical angle measured along the air gap in the frame of one pole-pair. In the case b) the density has a form of the rectangular pulses having the width of the slot. In the case c) the density has a form of the Dirac pulses $\delta(\alpha)$ located to the slot. The case d) deals with rectangular pulses centered to the slot center.

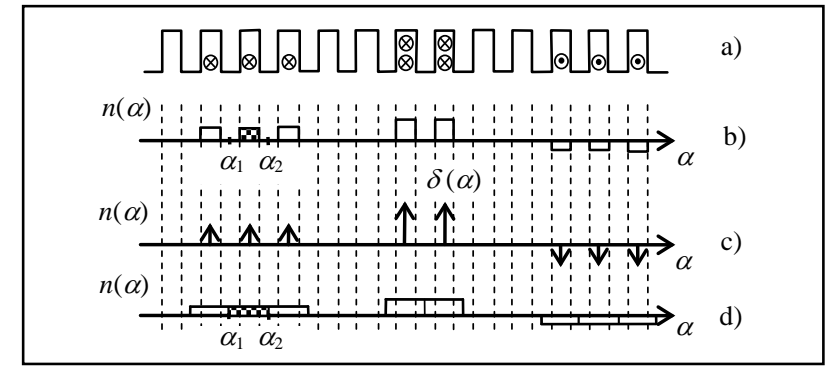

Fig. 1. Explanation of the notation of conductors density $n(\alpha)$.

The local density of conductors $n(\alpha)$ is defined as:

$$
n(\alpha)=\frac{\mathrm{d} N(\alpha)}{\mathrm{d} \alpha}
$$

\section{FLUX DENSITY AND LINKAGE FLUX}

Fig. 2. shows the quadrant of one pole-pair of the induction motor.

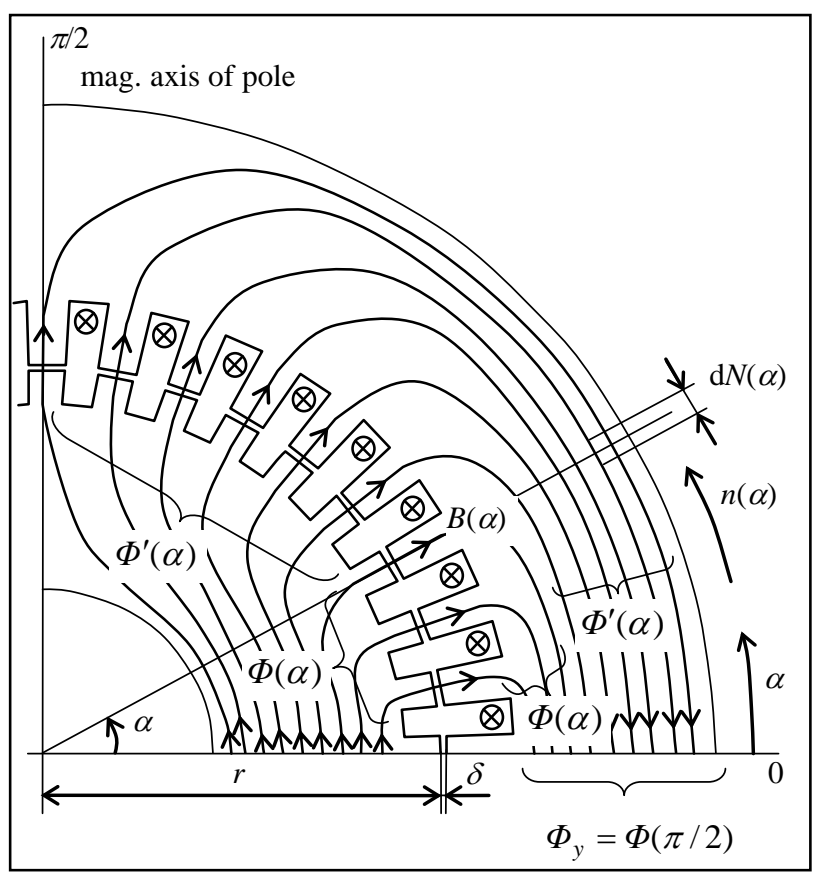

Fig. 2. Quadrant of one pole-pair of the induction motor

From the known distribution $n(\alpha)$ of conductors we may determine function $B(\alpha)$ according to Fig. 2:

$$
B(\alpha)=I_{\mu} \frac{\mu_{0}}{\delta} \int n(\alpha) \mathrm{d} \alpha,
$$

where $I_{\mu}$ is the magnetization current, $\delta$ is length of the air gap, 
and $\mu_{0}$ is permeability of free space. With help of equation (3), it is possible to determine equations for the linkage flux $\Psi$ of the whole pole-pair:

$$
\Psi_{\mathrm{x}, \mathrm{y}}=\frac{\operatorname{lr}}{p} \int_{0}^{2 \pi}[\underbrace{\int_{\begin{array}{l}
\alpha \\
\text { winding } n_{\mathrm{y}}(\alpha)
\end{array}}^{2 \pi} B_{\mathrm{x}}(\alpha) \mathrm{d} \alpha}_{\begin{array}{l}
\text { flux } \phi_{x}^{\prime}(\alpha) \text { linkaged } \\
\text { with winding } n_{\mathrm{y}}(\alpha)
\end{array}} \underbrace{n_{\mathrm{y}}(\alpha)}_{\begin{array}{c}
\text { inkaged with } \\
\text { flux } \phi_{x}^{\prime}(\alpha)
\end{array}} \mathrm{d} \alpha
$$

where $p$ is number of pole-pairs.

The equation may be used to electromagnetic design: a) the synchronous motor with the PM on the rotor - the field $B_{\mathrm{x}}(\alpha)$ of the permanent magnets affects the winding $n_{\mathrm{y}}(\alpha)$. b) the induction motor - the whole field $B_{\mathrm{x}}(\alpha)$ excited by a few windings affects the winding $n_{\mathrm{y}}(\alpha)$.

\section{ELECTROMAGNETIC DESIGN OF AC MACHINES ON THE FIRST HARMONIC}

The function $n(\alpha)$, i.e. $B(\alpha)$, can be evolved in the form of the Fourier series, see (Folland, 1992):

$$
n(\alpha)=\sum_{k=1}^{\infty}\left[a_{k} \cos (k \alpha)+b_{k} \sin (k \alpha)\right],
$$

where

$$
\begin{aligned}
& a_{k}=\frac{1}{\pi} \int_{0}^{2 \pi} n(\alpha) \cos (k \alpha) d \alpha, \\
& b_{k}=\frac{1}{\pi} \int_{0}^{2 \pi} n(\alpha) \sin (k \alpha) d \alpha .
\end{aligned}
$$

It is good to introduce new variable $n_{\mathrm{k}}$ for magnitude of $k$ harmonic component of density $n(\alpha)$ :

$$
n_{k}=\sqrt{a_{k}^{2}+b_{k}^{2}}
$$

With help of (8) is possible to rewrite (4), in case $x=y$, into form:

$$
\Psi=\sum_{k=1}^{\infty} \Psi_{k}=\pi \frac{\mu_{0} l r I_{\mu}}{p \delta} \sum_{k=1}^{\infty} \frac{n_{k}^{2}}{k} .
$$

For the winding design, it is necessary to use only the amplitude of the first harmonic $\Psi_{1}$. Let the whole phase motor winding be supplied by the harmonic voltage with the amplitude $U_{\mathrm{a}}$ :

$$
\Psi(t)=\int \frac{U_{\mathrm{a}}}{p} \cos (\omega t) \mathrm{d} t=\frac{U_{\mathrm{a}}}{\omega p} \sin (\omega t)=\Psi_{1} \sin (\omega t) .
$$

By comparison of the magnitude of the first harmonic of the linkage flux in equations (9) and (10) we get the amplitude $n_{1}$ of the useful first harmonic density of one pole-pair:

$$
n_{1}=\frac{U_{r m s}}{\sqrt{2} \pi^{2} f B_{\text {max }} l r} .
$$

\section{WINDING DESIGN PROCEDURE}

a) We choose the winding type, i.e. we choose the actual function $n(\alpha)$ under one pole-pair without knowing the total number $N$ of conductors. That means we consider the function $n(\alpha)$ with the relative unknown height $n_{0}$. b) The number $N$ of conductors must suit the equation:

$$
2 N=\int_{0}^{2 \pi}|n(\alpha)| \mathrm{d} \alpha .
$$

From this equation, it is possible to determine the explicit relation between the height $n_{0}$ and number $N$. We get the absolute function $n(\alpha)$ expressed with the help of number $N$.

c) The absolute function $n(\alpha)$ will be evolved to Fourier series from which we find the amplitude $\mathrm{n} 1$ of the first harmonic.

d) We compare the amplitude $n_{1}$ with the right side of the equation (9). In this way we determine a searched total number $N$ of conductors.

e) We recalculate the total number $N$ of conductors into individual slots according to the absolute function $n(\alpha)$. To this purpose, we use the geometrical interpretation according to Fig. 1. d). In one slot, marked in the figure, the number $N_{\mathrm{s}}$ of conductors will be:

$$
N_{s}=\int_{\alpha_{1}}^{\alpha_{2}} n(\alpha) \mathrm{d} \alpha
$$

where the interval $\left\langle\alpha_{1} ; \alpha_{2}\right\rangle$ is equal to the slot pitch $\alpha_{\mathrm{s}}$ and the interval center lies in the slot center. We use the equation (13) similarly for all the slots within a pole. The total distribution of conductors to slots is checked according to the following equation:

$$
N=\sum_{-\pi / 2}^{\pi / 2} N_{s}
$$

Thus, the winding design has been completed.

\section{CONCLUSION}

The aim of the paper has been to show a new method of electromagnetic design of AC machines, i.e. design of AC machines windings.

The new method can also be used for direct calculation:

- $\quad$ the flux density in the air gap.

- $\quad$ the linkage flux of the winding.

- the self and mutual inductance of the winding

The new method can be used for electromagnetic design of:

- $\quad$ the induction machines.

- $\quad$ the synchronous machines with permanent magnets.

\section{ACKNOWLEDGEMENTS}

This work was solved in the frame of the faculty project FEKT-S-11-14; of the project MSM 0021630516; and of project MPO FR-TI1/061; and project OP VK CZ.1.07/2.3.00/09.0162.

\section{REFERENCES}

Boldean I.; Nasar S. A. (2000). The Induction Machine Handbook, CRC Press, ISBN 9780849300042, London

Folland G. B. (1992). Fourier Analysis and Its Application, Thomson Brooks/Cole, ISBN 9780534170943, Pacific Grove

Langsdorf A. S. (1955). Theory of Alternating Current Machinery, McGraw-Hill, ISBN none, New York

Nowotny D. W.; Lipo T. A. (1996). Vector Control and Dynamics of AC Drives, Oxford University Press, ISBN 0198564392, New York

Pyrhonen J.; Jokinen T. \& Hrabovcova V. (2008). Design of rotating electrical machines, John Wiley \& Sons, ISBN 0470695166, Chichester 